\title{
Over-sampling algorithm for imbalanced data classification
}

\author{
XU Xiaolong ${ }^{1, *}$, CHEN Wen ${ }^{2}$, and SUN Yanfei ${ }^{3}$ \\ 1. Jiangsu Key Laboratory of Big Data Security \& Intelligent Processing, Nanjing University of Posts and Telecommunications, \\ Nanjing 210023, China; 2. Institute of Big Data Research at Yancheng, Nanjing University of Posts and Telecommunications, \\ Yancheng 224000, China; 3. Office of Scientific R\&D, Nanjing University of Posts and Telecommunications, Nanjing 210023, China
}

\begin{abstract}
For imbalanced datasets, the focus of classification is to identify samples of the minority class. The performance of current data mining algorithms is not good enough for processing imbalanced datasets. The synthetic minority over-sampling technique (SMOTE) is specifically designed for learning from imbalanced datasets, generating synthetic minority class examples by interpolating between minority class examples nearby. However, the SMOTE encounters the overgeneralization problem. The densitybased spatial clustering of applications with noise (DBSCAN) is not rigorous when dealing with the samples near the borderline. We optimize the DBSCAN algorithm for this problem to make clustering more reasonable. This paper integrates the optimized DBSCAN and SMOTE, and proposes a density-based synthetic minority over-sampling technique (DSMOTE). First, the optimized DBSCAN is used to divide the samples of the minority class into three groups, including core samples, borderline samples and noise samples, and then the noise samples of minority class is removed to synthesize more effective samples. In order to make full use of the information of core samples and borderline samples, different strategies are used to over-sample core samples and borderline samples. Experiments show that DSMOTE can achieve better results compared with SMOTE and Borderline-SMOTE in terms of precision, recall and F-value.
\end{abstract}

Keywords: imbalanced data, density-based spatial clustering of applications with noise (DBSCAN), synthetic minority oversampling technique (SMOTE), over-sampling.

DOI: $10.21629 /$ JSEE.2019.06.12

\section{Introduction}

Imbalanced datasets refer to datasets whose numbers of samples in each class are not even. Imbalanced datasets exist in many real-world domains, such as network intrusion detection [1], spam detection [2], text classification [3],

Manuscript received June 25, 2018.

*Corresponding author.

This work was supported by the National Key Research and Development Program of China (2018YFB1003700), the Scientific and Technological Support Project (Society) of Jiangsu Province (BE2016776), the “333" project of Jiangsu Province (BRA2017228; BRA2017401), and the Talent Project in Six Fields of Jiangsu Province (2015-JNHB-012). and medical application [4]. In these domains, what we are really interested in is the minority class other than the majority class. The solutions to imbalanced datasets can be divided into data and algorithmic levels. The methods at the data level change the distribution of the imbalanced datasets, and then the balanced datasets are provided to the learner to improve the detection rate of the minority class. The methods at the algorithm level modify the existing data mining algorithms or put forward new algorithms to resolve the imbalance problem. There are still some problems with these methods: (i) The methods at the algorithm level include cost sensitive learning methods $[5,6]$ and ensemble learning methods $[7,8]$. For datasets with different characteristics, the applicable algorithms are different and do not have universal applicability. (ii) At the data level, the synthetic minority over-sampling technique (SMOTE) algorithm proposed by Chawla et al. [9] is a classical over-sampling algorithm, many scholars propose improved algorithms based on SMOTE, such as the Borderline-SMOTE algorithm [10], however, SMOTE and the current improved algorithms are all based on the $k$ nearest neighbor algorithm. These algorithms generally have high time complexity, are susceptible to noise data, and cannot make good use of the information of majority samples.

Aiming at these problems, we combine the optimized density-based spatial clustering of applications with noise (DBSCAN) [11] with the SMOTE to propose a densitybased SMOTE (DSMOTE). First, we use the optimized DBSCAN to classify the minority samples into three groups which are core samples, borderline samples and noise samples; then, the noise samples of the minority class are removed to synthesize more effective samples; finally, the information of core samples and borderline samples, oversampling core samples and borderline samples is made full use of according to different strategies.

This paper is organized as follows. Section 2 introduces 
the related works to solve the imbalance problem. Section 3 introduces the proposed DSMOTE. Section 4 compares our method with other typical over-sampling methods. Section 5 draws conclusions and presents our future work.

\section{Related work}

\subsection{Methods at the algorithm level}

For handling the problem of the classification of imbalanced datasets, the methods at the algorithm level modify the existing data mining algorithms or put forward new algorithms to resolve the imbalance problem [12]. Chawla et al. [6] proposed a novel approach for learning from imbalanced datasets, based on a combination of the SMOTE algorithm and the boosting procedure. Unlike standard boosting that all misclassified examples are given equal weights, this algorithm creates synthetic examples from the rare or minority class, thus indirectly changing the updating weights and compensating for skewed distributions. The traditional algorithm, Ada-boost [7], proposed by Freund et al., is used to reduce the error of the algorithm that consistently generates classifiers. Its performance is a little better than random guessing. Based on the AdaBoost algorithm, Fan et al. [8] proposed a variant algorithm, which uses the cost of misclassifications to update the training distribution on successive boosting rounds. Joshi et al. [13] proposed an enhanced algorithm which brings an extra capability for achieving better balance between recall and precision in data mining. Wu et al. [14] proposed the class-boundary alignment algorithm to augment support vector machines (SVMs) to deal with imbalanced training data problems posed by emerging applications, which works effectively with images and video sequences. Huang et al. [15] proposed a biased minimax probability machine (BMPM) algorithm. Given the reliable mean and covariance matrices of the majority and minority classes, BMPM can derive the decision hyperplane by adjusting the lower bound of the real accuracy.

\subsection{Methods at the data level}

At the data level, the typical resampling methods include the random over-sampling and the random undersampling. The random under-sampling may reduce some useful information of the datasets. For example, Tomek et al. [16] proposed a method named Tomek links, which can be defined as a pair of minimally distanced nearest neighbors of opposite classes. If two samples are from one Tomek link, each of them is noise or both are near a border, which can be used to under-sample and remove samples belonging to majority classes in Tomek links. The random over-sampling may make the decision regions of the learner smaller and more specific, which causes the learner to over-fit. SMOTE creates synthetic minority class examples by interpolating between minority class examples that are close together. However, SMOTE encounters the overgeneralization problem. It generalizes the region of a minority class blindly without considering the majority class.

Some improved re-sampling methods were proposed to solve problems of the above algorithms. Sáez et al. [17] proposed the extension of SMOTE with an iterative ensemble-based noise filter called iterative-partitioning filter (IPF), which can overcome the problems generated by noisy and borderline examples in imbalanced datasets. Ma et al. [18] proposed a scheme for the imbalanced data classification problem, that the combination of clustering using representatives (CURE) enhances the SMOTE algorithm effectively compared with Borderline-SMOTE. In order to expand the decision regions, Dong et al. [19] proposed an over-sampling algorithm named Random-SMOTE. Different from SMOTE to synthesize a new sample on the line between two samples, for each sample $x$ of the minority class, two samples $y_{1}, y_{2}$ are randomly selected from the minority class, so a triangle is formed by $x, y_{1}$ and $y_{2}$; then, a sample $t_{x}$ is selected along the line between $y_{1}$ and $y_{2}$; finally a new synthetic sample is generated along the line between $t_{x}$ and $x$. Han et al. [10] proposed BorderlineSMOTE, which only oversamples or strengthens the borderline minority samples. The Borderline-SMOTE algorithm classifies the samples of the minority class into three groups which are safe samples, dangerous samples and noise samples. Dangerous samples indicate that more than half of the $m$ nearest neighbors of this minority sample are the majority samples; safe samples indicate that more than half of the $m$ neighbors of the minority sample are minority samples; and noise samples indicate that $m$ neighbors are majority samples. The borderline examples of the minority class are more easily misclassified than those ones far from the borderline. Borderline-SMOTE performs better than SMOTE, while Borderline-SMOTE only oversamples the dangerous points and does not utilize the information of safe samples. Haibo et al. [20] proposed adaptive synthetic sampling approach for imbalanced learning (ADASYN). ADASYN uses a weighted distribution for different minority class examples according to their level of difficulty in learning. More synthetic data are generated for minority class examples harder to learn compared to those minority examples easier to learn. Bunk-humporn-pat et al. [21] proposed an algorithm to generate synthetic samples along a shortest path from each positive sample to a pseudo centroid of a minority-class cluster, which is different from the algorithm proposed in this paper. Our method oversamples core samples and borderline samples according to different strategies. Gutiérrez et al. [22] combined graph- 
ics processing unit (GPU) devices with a proper data handling scheme in order to perform data sampling algorithms based on SMOTE to deal with large datasets on a wide variety of commodity hardware, including laptop computers. Zhou et al. [23] proposed a new SMOTE, namely the misclassification cost minimization oriented SMOTE (CMOSMOTE) to achieve the satisfactory performance. Zhang et al. [24] proposed an over-sampling method for imbalanced data classification to achieve better performance compared with SMOTE and borderline-SMOTE. To solve the problem of the low detection rate of minority samples in imbalanced datasets in network intrusion detection. Zhang et al. [25] proposed a deep learning-based intrusion detection model based on optimized imbalanced data. SMOTE was used to increase the number of samples in minority categories and the majority of the samples were under-sampled by neighborhood cleaning rule (NCL) in data processing. Jiang et al. [26] proposed a novel genetic algorithm based on SMOTE, which uses different sampling rates for different minority class instances and finds the combination of optimal sampling rates. Prusty et al. [27] proposed a weighted-SMOTE (WSMOTE). Oversampling of each minority data sample is carried out based on the weight assigned to it. These weights are determined by using the Euclidean distance of a particular minority data sample with respect to all the remaining minority data samples. Each minority data sample need not generate an equal number of synthetic data in WSMOTE as in the case of SMOTE. Gong et al. [28] proposed a novel classification approach to solve the online data imbalance problem by integrating a fast and efficient learning algorithm. Xue et al. [29] proposed a novel approach for learning from imbalanced datasets by organically combining the over-sampling and the under-sampling method, which aims to choose targeted neighbors and synthesize samples with different strategies. Su et al. [30] proposed an improved SMOTE and the XGBoost intrusion detection method to oversample for the class with a small amount of data and downsample for a class with a large amount of data to achieve high accuracy and low missing rate in intrusion detection. Bhagat et al. [31] introduced a methodology for classification of multi-class imbalanced data with two steps. Demidova et al. [32] proposed a new approach to select optimal parameters values for the SMOTE algorithm. Junsomboon et al. [33] proposed a solution to adjust imbalance dataset by combining the NCL and SMOTE. Gosain et al. [34] proposed an algorithm to generate synthetic samples along the line joining the minority samples and its $k$ minority class farthest neighbors. Sun et al. [35] proposed a new ensemble model for imbalanced enterprise credit evaluation based on SMOTE and the Bagging ensemble learning algorithm with differentiated sampling rates (DSR). Harli- man et al. [36] proposed a hybrid method to improve imbalanced set performance by tuning weights for each class, where oversampling starts from borders, the ripple moves toward the centroid, and then synthetic samples are generated based on the nearest neighbor sample from the ripples.

\section{DSMOTE}

\subsection{DBSCAN algorithm optimization}

DBSCAN is used to discover clusters of arbitrary shape. Related definitions of DBSCAN are as follows.

Definition 1 (Eps-neighborhood of a point) The Epsneighborhood of a point $q$, denoted by $N_{E p s}(q)$, is defined as

$$
N_{E p s}(q)=\{p \in D \mid \operatorname{dist}(p, q) \leqslant E p s\}
$$

where $\operatorname{dist}(p, q)$ represents the distance between object $p$ and object $q$.

Definition 2 (Directly density-reachable) Let $D$ be a database of points. Given Eps and MinPts, $p$ and $q$ are two objects in dataset $D . p$ is directly density-reachable from $q$, satisfying the following conditions:

$$
\begin{gathered}
p \in N_{\text {Eps }}(q) \\
\left|N_{\text {Eps }}(q)\right| \geqslant \text { MinPts. }
\end{gathered}
$$

Definition 3 (Density-reachable) Given Eps and MinPts, if there is a chain of points $p_{1}, \ldots, p_{n}, p_{1}=q$, $p_{n}=p, p_{i+1}$ is directly density-reachable from $p_{i}$.

Definition 4 (Density-connected) If there is a point $r$, both $p$ and $q$ are density-reachable from $r$.

Definition 5 (Cluster) Suppose that $A$ cluster $C$ is a non-empty subset of $D$ satisfying the following conditions:

(i) $\forall p, q \in D$ : if $p \in C$ and $q$ is density-reachable from $p$, then $q \in C$ (Maximality).

(ii) $\forall p, q \in C: p$ is density-connected to $q$.

According to Definition 1, $\operatorname{dist}(p, q)$ is the distance between $p$ and $q$. According to Definition 2, if $p$ is directly-density to $q, q$ is a core point and $p$ in the Epsneighborhood of $q$. According to Definition 3, the maximum density-connected point sets can be derived from the density-reachable relationship.

DBSCAN has a problem that if cluster $C_{1}$ and cluster $C_{2}$ are relatively close, some points at the junction of the two clusters may be density connected to core points of the different clusters at the same time. Assume that object $q_{1}$ is a core point in cluster $C_{1}, q_{2}$ is a core point in cluster $C_{2}$, and point $u$ is density-reachable to $p$ and $q$. If DBSCAN expands $q_{1}$ first, then object $u$ belongs to $C_{1}$; otherwise, $u$ belongs to $C_{2}$, thus the attribution of some borderline points depends on the order of core points traversing. Therefore, DBSCAN is not rigorous enough to deal with the boundary objects of clusters. 
For above problems we propose a solution based on $k$ means. In $k$-means, points belong to the cluster of the nearest centroid. If there are core points of different classes in the Eps neighborhood of non-core point $p$, calculate the "center" of clusters belonging to these different classes of core points, then compute the Euclidean distance between $p$ and these "centers", and find the nearest center to $p$.

Each cluster can be represented by its center [37]. The core point is closer to the center of the cluster than borderline samples. Therefore, we can calculate the centers of clusters based on these core points. Compared with the $k$-means algorithm, each iteration uses all the points to calculate the centroid of the clusters. We calculate the center of the clusters according to these core points without noise, so that the clustering is more reasonable.

Definition 6 (Cluster center) Supposing that each core sample has $r$ features, the number of core samples is $n$, and the core sample set is labeled as $C, C=\left\{c_{1}, c_{2}, \ldots, c_{n}\right\}$, the cluster center is calculated as follows:

$$
C_{\text {center }}=\left(\frac{1}{n} \sum_{i=1}^{n} c_{i 1}, \frac{1}{n} \sum_{i=1}^{n} c_{i 2}, \ldots, \frac{1}{n} \sum_{i=1}^{n} c_{i r}\right) .
$$

\subsection{DSMOTE}

Inspired by Borderline-SMOTE, we propose DSMOTE. DSMOTE not only oversamples borderline samples, but also oversamples the "safe" sample. First, we use the optimized DBSCAN to classify the samples of the minority class into three groups which are core samples, borderline samples and noise samples, and then remove the noise samples of the minority class to synthesize more effective samples. In order to make full use of the information of core samples and borderline samples, we use different strategies to over-sample core samples and borderline samples. The workflow of DSMOTE is shown in Fig. 1.

Specific steps of DSMOTE are as follows:

(i) Use the optimized DBSCAN to classify the samples of the minority class into three groups including core samples, borderline samples and noise samples, and then remove the noise samples of the minority class.

Input: Original dataset T, MinPts, Eps, and the number of minority samples $T_{\min }$

Output: Synthetic minority class samples $\left(N \% * T_{\min }\right)$

i) Select an unprocessed sample $p$ from $T$.

ii) If $p$ is a core point, a new cluster is generated, then expand $p$, that is, find all density connected samples. Core samples are assigned to the cluster, and all non-core samples are marked as undetermined borderline samples. If $p$ is not a core sample, mark $p$ as a noise.

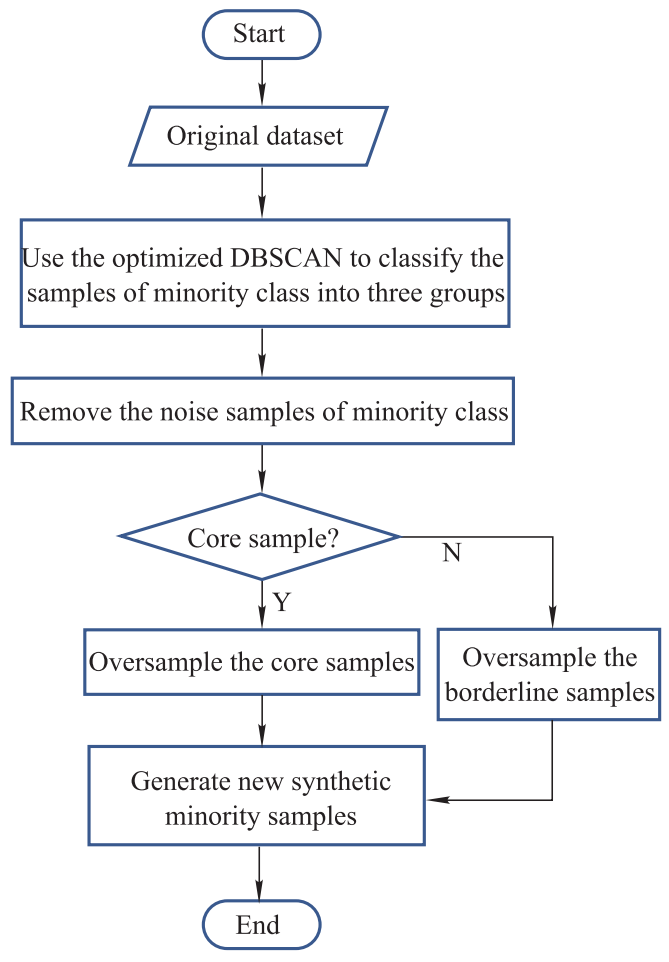

Fig. 1 Workflow of DSMOTE

iii) In order to discover other clusters, the dataset needs to be rescanned. If there are unprocessed samples in $T$, jump to i).

iv) We divide the undetermined borderline samples. For the borderline sample $u$, if all the core points in its Eps neighborhood belong to the same cluster, $u$ belongs to the cluster of these core points; if there are two core points $q_{1}$ and $q_{2}$ in the Eps neighborhood belonging to different clusters, we calculate the Euclidean distance $D_{1}$ from the center of $q_{1}$ to $u$, and the Euclidean distance $D_{2}$ from the center of $q_{2}$ to $u$. If $D_{1}<D_{2}, u$ is divided into the center of $q_{1}$; otherwise divided into the center of $q_{2}$.

v) Remove the minority data labeled as noise. Points that are not included in any cluster are noise, that is, points that do not belong to any cluster, and equivalence can be defined as density not reachable from any one of the core points. In order to make the synthesized new samples more reasonable and effective, we remove the minority data labeled as noise.

Suppose that $C$ denotes the core sample set, $B$ denotes the borderline sample set, $C=\left\{c_{1}, c_{2}, \ldots, c_{k}\right\}$, $B=\left\{b_{1}, b_{2}, \ldots, b_{m}\right\}, k$ is the number of samples in $C$, and $m$ is the number of samples in $B$.

(ii) Oversample the core samples. For every $c_{i}(i=$ $1,2, \ldots, k)$ in the minority core samples $C, N$ samples are randomly selected from its Eps neighborhood. The difference $d_{j}(j=1,2, \ldots, N)$ between $c_{i}$ and selected samples from Eps neighborhood are calculated, where $d_{j}$ 
is the vector difference between each feature of the randomly selected sample minus the corresponding feature of the core sample $c_{i}$; select a random number $r_{j}$ from 0 to 1 . Then multiply $d_{j}$ by $r_{j}$ to get a synthetic sample $s_{j}$, and $N$ new synthetic minority samples are generated between $c_{i}$ and selected samples:

$$
s_{j}=c_{i}+r_{j} \times d_{j}, \quad j=1,2, \ldots, N .
$$

Repeat the above steps for each sample $c_{i}$ in $C$, and finally $k \times N$ new samples can be synthesized.

(iii) Oversample the borderline samples. First, we calculate the cluster center $C_{\text {center }}$ of minority samples according to Definition 6. Then for each sample $b_{i}$ in $B$, calculate the difference $d_{j}$ between $b_{i}$ and $C_{\text {center }}, d_{j}$ is the vector difference between each feature of the sample $b_{i}$ minus the corresponding feature of the center $C_{\text {center }}$; select a random number $r_{j}$ from 0 to 1 , and then multiply $d_{j}$ by $r_{j}$ to get a synthetic sample $s_{j}$. Repeat these steps, $N$ new synthetic minority samples are generated between $b_{i}$ and $C_{\text {center }}$ :

$$
s_{j}=C_{\text {center }}+r_{j} \times d_{j}, \quad j=1,2, \ldots, N .
$$

Repeat the above steps for each sample $b_{i}$ in $B$, and finally $m \times N$ new samples can be synthesized.

From the analysis above, it can be seen that DSMOTE is different from the DBSMOTE algorithm. DBSMOTE generates synthetic samples along a shortest path from each positive sample to a pseudo centroid of a minority-class cluster. DBSMOTE has high time complexity. To make full use of the information of core samples and borderline samples, DSMOTE uses different strategies to over-sample core samples and borderline samples.

\subsection{Computational complexity}

The computational complexity of SMOTE is $O\left(n \log _{2} n\right)$. SMOTE finds the $k$ nearest neighbor samples and the new synthesized samples for each minority sample, which are $O(n)$ and $O(1)$, Borderline-SMOTE is based on SMOTE. Therefore, its complexity is also $O\left(n^{2}\right)$, where $n$ is the number of borderline samples. The complexity of DBSMOTE is $O\left(n^{2}\right)$ [21], where $n$ is the number of minority samples.

The complexity of our proposed DSMOTE is $O\left(n \log _{2} n\right)$. The calculation time of the optimized DBSCAN is divided into two parts. The first part $T_{1}(n)$ is the division of the core points and the calculation time is $O\left(n \log _{2} n\right)$. The second part $T_{2}(n)$ is the division of borderline samples, and the calculation time is $O\left(n \log _{2} n\right)$. The cluster center and the distance from the cluster center are calculated for minority borderline samples. The second part of the calculation time is $O\left(n \log _{2} n\right)$. The minority core samples and borderline samples are oversampled. The calculation time $T_{3}(n)$ is $O(n)$. That is why the complexity $T(n)$ of DSMOTE is $O\left(n \log _{2} n\right)$.

\section{Experiments}

\subsection{Evaluation metrics}

For imbalanced datasets, the performance of a classifier is not only measured by the classification accuracy. The multi-class problem can be simplified to the two-class problem: the class label of the minority class is positive, and the class label of the majority class is negative. Precision, Recall and F-value as evaluation metrics of the two-class problem are more reasonable for imbalanced dataset learning. Table 1 shows a confusion matrix of a two-class problem. The first column of the table is the actual class label of the samples. TP and $T N$ denote the number of positive and negative samples that are classified correctly, while $F N$ and FP denote the number of misclassified positive and negative samples respectively.

Table 1 Confusion matrix for the two-class problem

\begin{tabular}{ccc}
\hline Actual label & Predicted positive & Predicted negative \\
\hline Positive & $T P$ & $F N$ \\
Negative & $F P$ & $T N$ \\
\hline
\end{tabular}

Precision indicates the ratio of positive samples that are classified correct to samples that are classified as positive, as follows:

$$
\text { Precision }=\frac{T P}{T P+F P} .
$$

Recall indicates the ratio of positive samples that are classified correct to actual positive samples, as follows:

$$
T \text { Prate }=\text { Recall }=\frac{T P}{T P+F N} .
$$

F-value incorporates both Precision and Recall, as follows:

$$
\mathrm{F} \text {-value }=\frac{\left(1+\beta^{2}\right) \cdot \text { Precision } \cdot \text { Recall }}{\beta^{2} \cdot \text { Precision }+ \text { Recall }} .
$$

\subsection{Experimental environment and datasets}

The hardware platform of our experiments includes $8 \mathrm{G}$ RAM, Intel core i5 processor at $1.8 \mathrm{GHz}$, and $500 \mathrm{~GB}$ hard disk. The software platform is based on WEKA and the implementation language is Java.

We collected four imbalanced datasets from the online UCI machine learning library [38]. Pima and Breast-w are two-class datasets, and the other two are multi-class datasets. We chose the smallest class or near the smallest class as the minority class. Table 2 shows the characteristics of the four datasets sorted by the imbalance level (IL) in ascending order, including the class label selected as minority class, the number of attributes (\#Attr), the number of samples in minority class (\#Minor), the number of samples in majority class (\#Major) and the IL. 
Table 2 Descriptions of four datasets

\begin{tabular}{cccccc}
\hline Dataset & Label & \#Attr & \#Minor & \#Major & IL \\
\hline Pima & 1 & 8 & 268 & 500 & 1.87 \\
Breast-w & 4 & 10 & 241 & 458 & 1.9 \\
Vehicle & 0 & 18 & 226 & 946 & 3.85 \\
Ecoli & 1 & 7 & 77 & 336 & 4.37 \\
\hline
\end{tabular}

Each dataset is divided into majority samples and minority samples. The IL is the ratio between the number of majority samples and the number of minority samples.

\subsection{Parameters}

In the predictor construction phase, we employ J48 classification algorithms. To evaluate the performance of these over-sampling methods, we perform $10 \times 10$ fold cross validation. The measurements of performance are averaged.

For different distributed data, if values of MinPts do not change, too large values of Eps will lead to too many points being gathered into the same cluster, while too small values of Eps will lead to the original cluster being divided into multiple clusters. If values of Eps do not change, too large values of MinPts will lead to too many points being marked as borderline points or noise, while too small values of MinPts will lead to too many points marked as core points.

Therefore, it is necessary to adjust Eps and MinPts to make the clustering effect better. We observe the performance of DSMOTE on Pima when different parameters are used in experiments. MinPts is set as 5 , and $N$ is set as $300 \%$. Experimental results show that Eps set in $[0.4,0.9]$ of the clustering effect is better. With the values of Eps in $[0.4,0.9]$, the change of F-value is shown in Fig. 2.

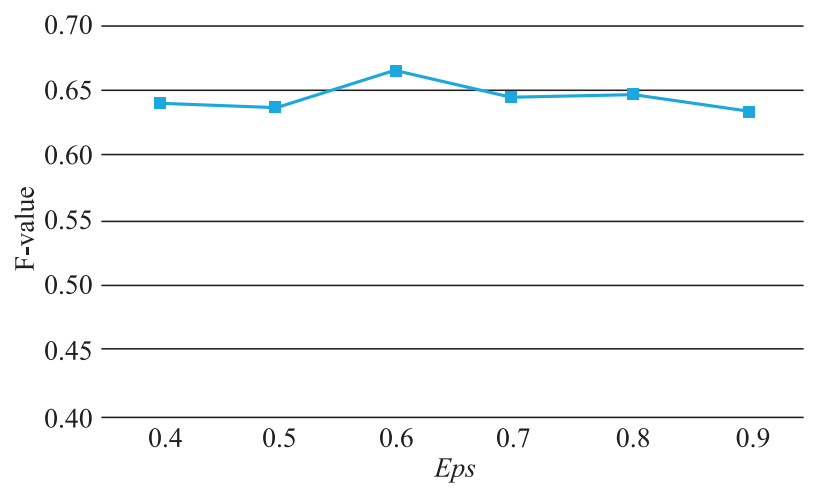

Fig. 2 F-value for the minority class of DSMOTE on Pima

\subsection{Experimental results and analysis}

The experimental results for all four datasets are showed in Table 3 to Table 6 and Fig. 3 to Fig. 14. The $x$-axis of these figures represents the over-sampling percent on the minority class. Precision, Recall and F-value for the minority class are reported by these tables and figures, while Precision, Recall and F-value of the majority class are not included. For each method, the best performance is highlighted in each category.

Analyzing Table 3 to Table 6, it is apparent that DSMOTE performs better than other methods. With same $N$, DSMTOE shows better Precision, Recall and F-value.

For Pima, DSMOTE achieves better performance obviously on Precision and F-value than both SMOTE and Borderline-SMOTE, with J48 applied as the classifier. SMOTE performs better on Precision with $N$ set higher than $300 \%$. Borderline-SMOTE only achieves a better performance on Recall with $N=100 \%$.

Table 3 Precision, Recall and F-value of minority class based on the Pima dataset

\begin{tabular}{ccccc}
\hline Method & $N / \%$ & Precision & Recall & F-value \\
\cline { 5 - 6 } \cline { 4 - 5 } Original & & 0.606 & 0.563 & 0.584 \\
\hline \multirow{3}{*}{ SMOTE } & 100 & 0.565 & 0.737 & 0.64 \\
& 200 & 0.547 & 0.768 & 0.639 \\
& 300 & 0.531 & 0.787 & 0.634 \\
& 400 & $\mathbf{0 . 5 3 3}$ & 0.813 & 0.643 \\
& 500 & $\mathbf{0 . 5 2 2}$ & 0.809 & 0.634 \\
\hline \multirow{2}{*}{ DSMOTE } & 100 & $\mathbf{0 . 5 7 4}$ & 0.737 & $\mathbf{0 . 6 4 6}$ \\
& 200 & $\mathbf{0 . 5 5 0}$ & $\mathbf{0 . 7 9 5}$ & $\mathbf{0 . 6 5}$ \\
& 300 & $\mathbf{0 . 5 3 7}$ & $\mathbf{0 . 8 1 5}$ & $\mathbf{0 . 6 4 7}$ \\
& 400 & 0.529 & $\mathbf{0 . 8 4 3}$ & $\mathbf{0 . 6 5}$ \\
Borderline- & 500 & 0.515 & $\mathbf{0 . 8 5 6}$ & 0.643 \\
\hline SMOTE & 100 & 0.545 & $\mathbf{0 . 7 6 3}$ & 0.636 \\
& 200 & 0.524 & 0.789 & 0.629 \\
& 300 & 0.509 & 0.791 & 0.619 \\
& 400 & 0.513 & 0.814 & 0.629 \\
& 500 & 0.504 & 0.803 & 0.643 \\
\hline
\end{tabular}

For Breast-w, DSMOTE, SMOTE and BorderlineSMTOE show similar performances on Precision, Recall and F-value. Borderline-SMOTE only achieves a better performance on Precision with $N=500 \%$, since the independent assumption on the borderline region is valid.

Table 4 Precision, Recall and F-value of minority class based on the Breast-w dataset

\begin{tabular}{|c|c|c|c|c|}
\hline Method & \multirow{2}{*}{$N / \%$} & Precision & Recall & $\bar{F}$-value \\
\hline Original & & 0.910 & 0.892 & 0.901 \\
\hline \multirow{5}{*}{ SMOTE } & 100 & 0.906 & 0.939 & 0.922 \\
\hline & 200 & 0.905 & 0.946 & 0.925 \\
\hline & 300 & 0.906 & 0.943 & 0.924 \\
\hline & 400 & 0.906 & 0.954 & 0.929 \\
\hline & 500 & 0.909 & 0.959 & 0.933 \\
\hline \multirow{5}{*}{ DSMOTE } & 100 & 0.913 & 0.953 & 0.932 \\
\hline & 200 & 0.906 & 0.954 & 0.929 \\
\hline & 300 & 0.909 & 0.953 & 0.930 \\
\hline & 400 & 0.909 & 0.954 & 0.931 \\
\hline & 500 & 0.910 & 0.963 & 0.935 \\
\hline \multirow{5}{*}{$\begin{array}{l}\text { Borderline- } \\
\text { SMOTE }\end{array}$} & 100 & 0.907 & 0.952 & 0.929 \\
\hline & 200 & 0.906 & 0.950 & 0.927 \\
\hline & 300 & 0.906 & 0.948 & 0.927 \\
\hline & 400 & 0.905 & 0.946 & 0.925 \\
\hline & 500 & 0.913 & 0.954 & 0.933 \\
\hline
\end{tabular}

For Vehicle, it is apparent that Precision, Recall and Fvalue are improved if the over-sampling percent on the minority class is increased. DSMOTE is superior to the other methods on all metrics. 
Table 5 Precision, Recall and F-value of minority class based on the Vehicle dataset

\begin{tabular}{ccccc}
\hline Method & \multirow{2}{*}{$N / \%$} & Precision & Recall & F-value \\
\cline { 1 - 1 } \cline { 4 - 5 } Original & & 0.874 & 0.874 & 0.874 \\
\hline \multirow{4}{*}{ SMOTE } & 100 & 0.890 & 0.894 & 0.892 \\
& 200 & 0.902 & 0.879 & 0.891 \\
& 300 & 0.881 & 0.854 & 0.867 \\
& 400 & 0.875 & 0.844 & 0.859 \\
& 500 & 0.887 & 0.829 & 0.857 \\
\hline \multirow{4}{*}{ DSMOTE } & 100 & $\mathbf{0 . 9 0 1}$ & $\mathbf{0 . 9 1 5}$ & $\mathbf{0 . 9 0 8}$ \\
& 200 & $\mathbf{0 . 9 0 5}$ & $\mathbf{0 . 9 1 0}$ & $\mathbf{0 . 9 0 7}$ \\
& 300 & $\mathbf{0 . 8 9 4}$ & $\mathbf{0 . 8 8 9}$ & $\mathbf{0 . 8 9 2}$ \\
& 400 & $\mathbf{0 . 9 1 6}$ & $\mathbf{0 . 8 7 4}$ & $\mathbf{0 . 8 9 5}$ \\
Borderline- & 500 & $\mathbf{0 . 9 0 1}$ & $\mathbf{0 . 8 6 4}$ & $\mathbf{0 . 8 8 2}$ \\
\hline SMOTE & 100 & 0.894 & 0.894 & 0.894 \\
& 200 & 0.890 & 0.854 & 0.872 \\
& 300 & 0.874 & 0.834 & 0.853 \\
& 400 & 0.887 & 0.864 & 0.875 \\
& 500 & 0.861 & 0.839 & 0.850 \\
\hline
\end{tabular}

For Ecoli, compared with SMOTE and BorderlineSMOTE, DSMOTE has a great improvement in Precision, Recall and F-value. In addition, Borderline-SMOTE shows the poor performance on higher percent.

Table 6 Precision, Recall and F-value of minority class based on the Ecoli dataset

\begin{tabular}{ccccc}
\hline Method & \multirow{2}{*}{$N / \%$} & Precision & Recall & F-value \\
\cline { 1 - 1 } \cline { 5 - 5 } Original & & 0.756 & 0.766 & 0.761 \\
\hline \multirow{4}{*}{ SMOTE } & 100 & $\mathbf{0 . 7 3 9}$ & 0.883 & 0.805 \\
& 200 & $\mathbf{0 . 7 3 4}$ & 0.896 & 0.807 \\
& 300 & 0.701 & 0.883 & 0.782 \\
& 400 & 0.693 & 0.909 & 0.787 \\
& 500 & 0.697 & 0.896 & 0.784 \\
\hline \multirow{5}{*}{ DSMOTE } & 100 & 0.737 & $\mathbf{0 . 9 0 9}$ & $\mathbf{0 . 8 1 4}$ \\
& 200 & 0.723 & $\mathbf{0 . 9 4 8}$ & $\mathbf{0 . 8 2 0}$ \\
& 300 & $\mathbf{0 . 7 1 1}$ & $\mathbf{0 . 8 9 6}$ & $\mathbf{0 . 7 9 3}$ \\
& 400 & $\mathbf{0 . 6 9 9}$ & $\mathbf{0 . 9 3 5}$ & $\mathbf{0 . 8 0 0}$ \\
Borderline- & 500 & $\mathbf{0 . 7 0 6}$ & $\mathbf{0 . 9 3 6}$ & $\mathbf{0 . 8 0 5}$ \\
\hline SMOTE & 100 & 0.697 & 0.896 & 0.784 \\
& 300 & 0.683 & 0.922 & 0.785 \\
& 400 & 0.66 & 0.882 & 0.756 \\
& 500 & 0.645 & 0.922 & 0.759 \\
& 0.642 & 0.909 & 0.753 \\
\hline
\end{tabular}

As shown in Fig. 3 to Fig. 14, their change tendencies are the same.

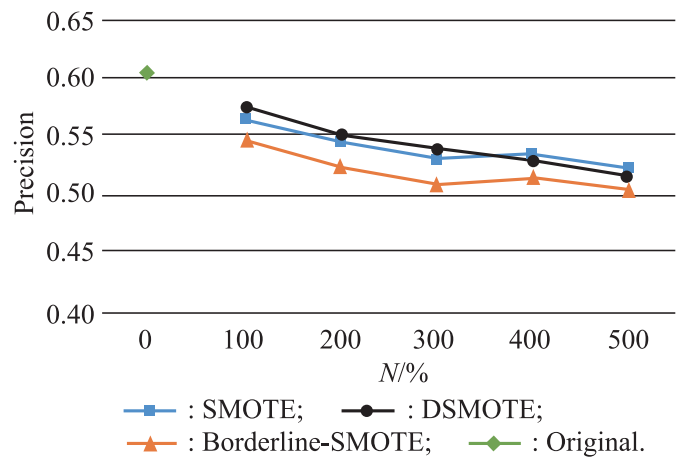

Fig. 3 Precision for the minority class as three methods applied on the Pima dataset

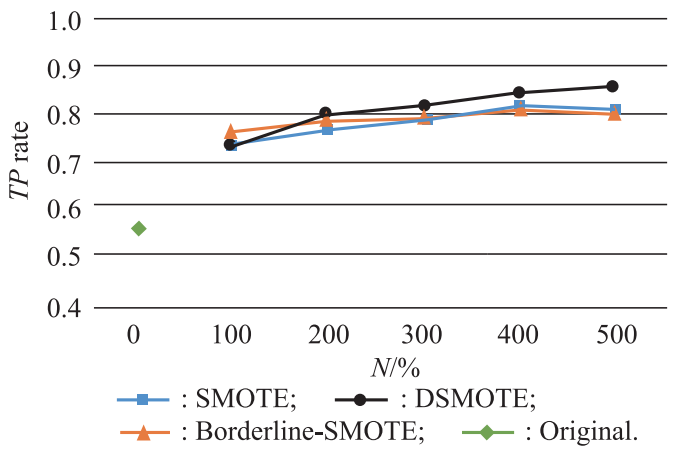

Fig. 4 Recall for the minority class as three methods applied on the Pima dataset

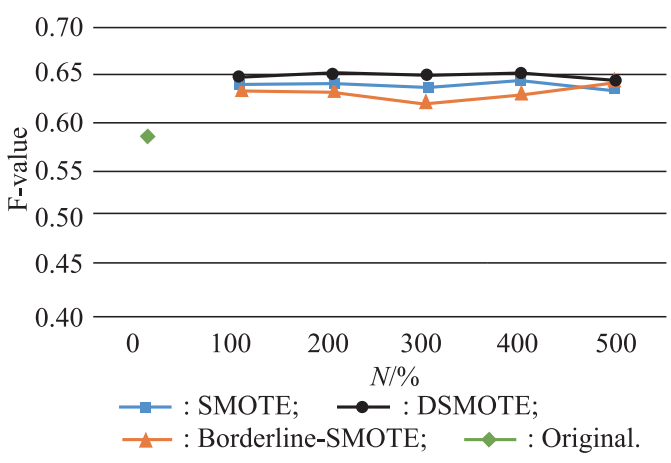

Fig. 5 F-value for the minority class as three methods applied on the Pima dataset

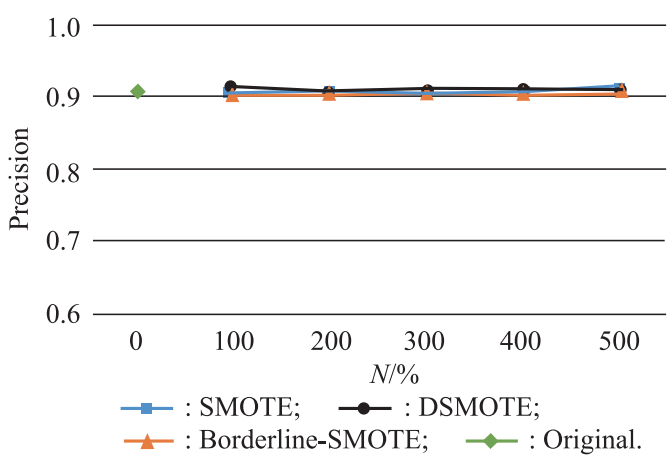

Fig. 6 Precision for the minority class as three methods applied on the Breast-w dataset

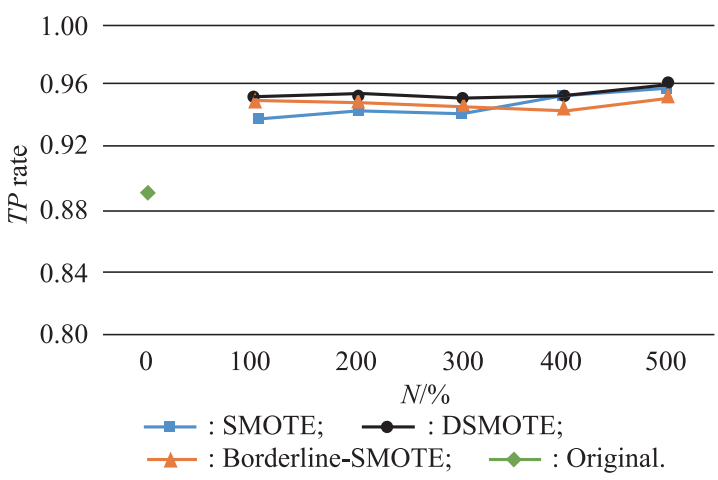

Fig. 7 Recall for the minority class as three methods applied on the Breast-w dataset 


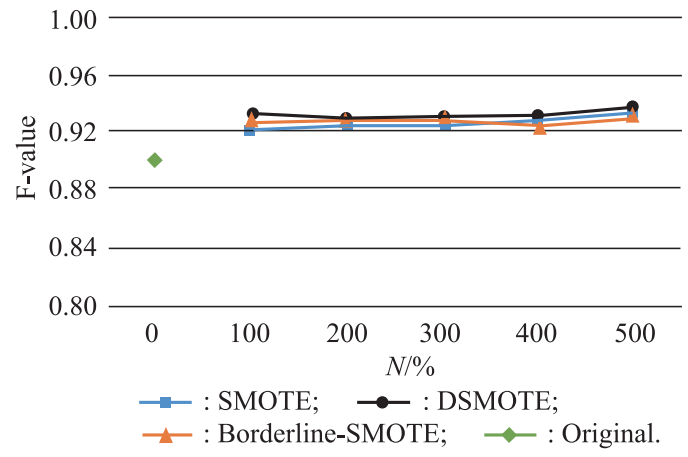

Fig. 8 F-value for the minority class as three methods applied on the Breast-w dataset

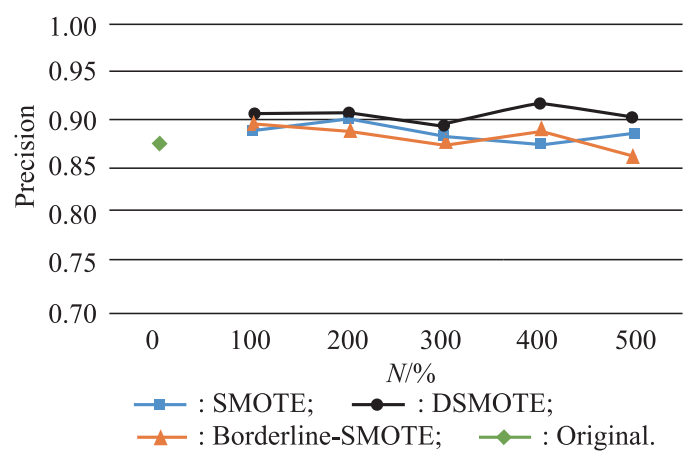

Fig. 9 Precision for the minority class as three methods applied on the Vehicle dataset

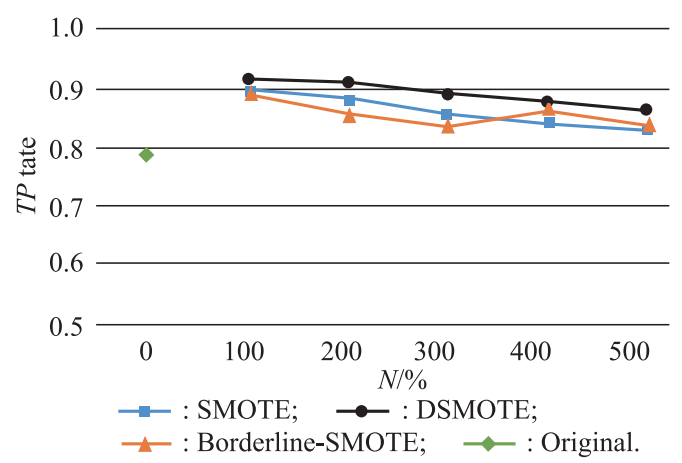

Fig. 10 Recall for the minority class as three methods applied on the Vehicle dataset

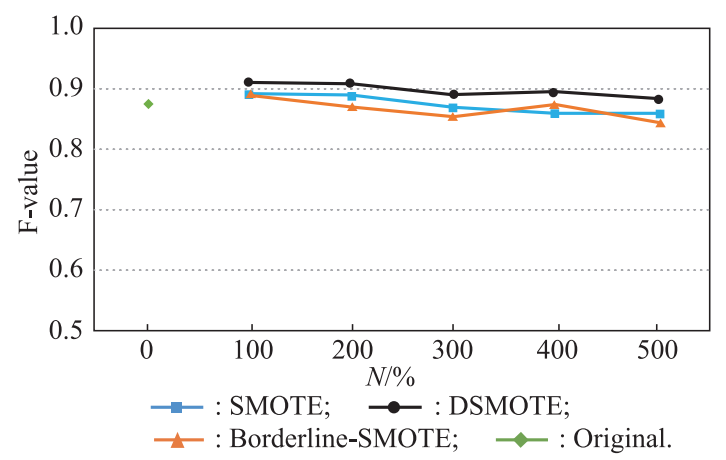

Fig. 11 F-value for the minority class as three methods applied on the Vehicle dataset

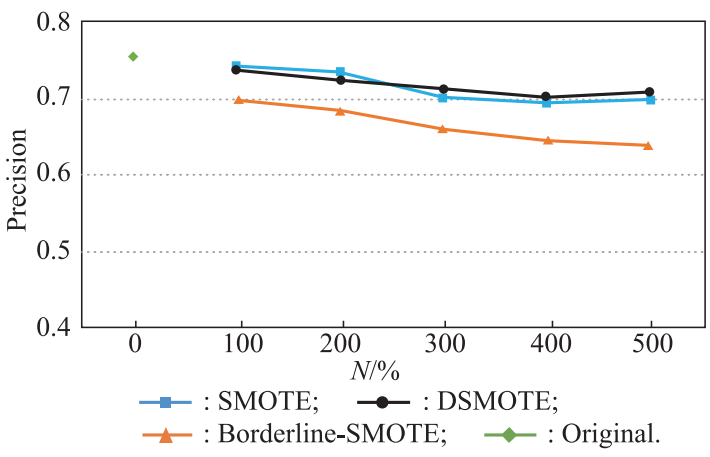

Fig. 12 Precision for the minority class as three methods applied on the Ecoli dataset

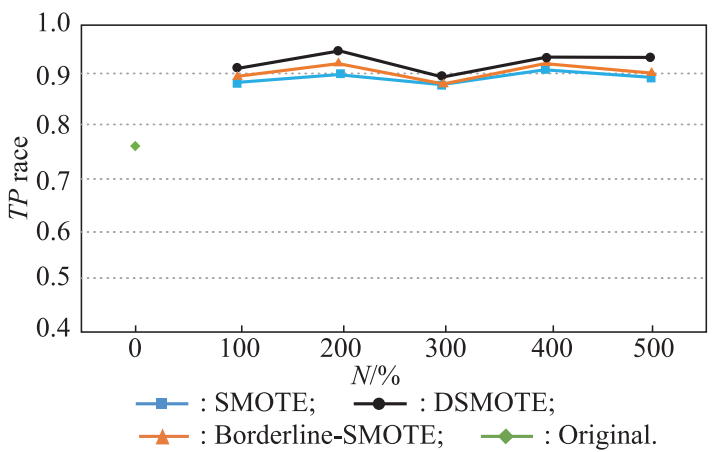

Fig. 13 Recall for the minority class as three methods applied on the Ecoli dataset

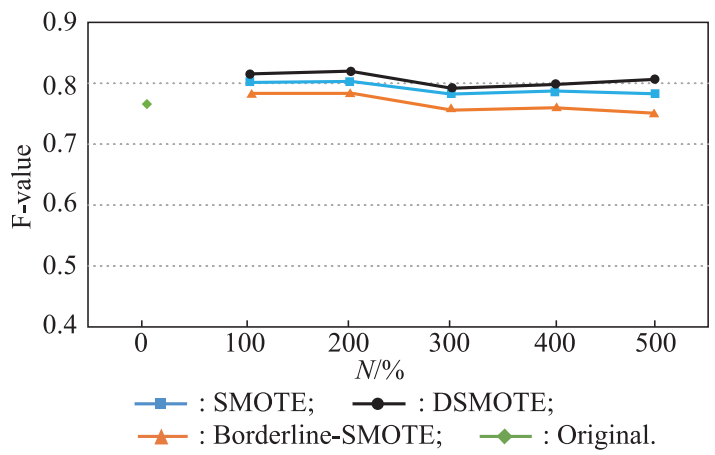

Fig. 14 F-value for the minority class as three methods applied on the Ecoli dataset

To sum up, the experiments provide strong evidences that DSMOTE is more suitable for the classification of imbalanced data sets. The reasons are shown as follows:

(i) DSMOTE eliminates the noise data of the minority class to make the synthesized samples more reasonable and effective.

(ii) DSMOTE makes full use of the information of core samples and borderline samples, oversampling core samples and borderline samples according to different strategies.

\section{Conclusions}

In order to solve the imbalance problem, we propose an oversampling method DSMOTE in this paper. We use the 
optimized DBSCAN to classify the samples of the minority class into three groups including core samples, borderline samples and noise samples. DSMOTE can not only eliminate the limitation of linear interpolation only among the positive samples, but also remove the noise in the dataset. We choose J48 as the classifier on four different datasets to test and compare the performances of DSMOTE, SMOTE and Borderline-SMOTE, which show that DSMOTE performs better. However, DSMOTE needs to manually set some parameters, which is not convenient enough. Our future work is to solve this problem to make DSMOTE more practical.

\section{References}

[1] TAN X P, SU S J, HUANG Z P, et al. Wireledss sensor networks intrusion detection based on SMOTE and the random forest algorithm. Sensors, 2019, 19(1): 203 .

[2] LI C L, LIU S G. A comparative study of the class imbalance problem in Twitter spam detection. Concurrency and Computation: Practice and Experience, 2017, 30(5): e4281.

[3] LI Y L, SUN G S, ZHU Y H. Data imbalance problem in text classification. Proc. of the 3rd International Symposium on Information Processing, 2010: 301-305.

[4] ZHU M, XIA J, JIN X Q, et al. Class weights random forest algorithm for processing class imbalanced medical data. IEEE Access, 2018, 6: $4641-4652$.

[5] WEI X. Research of ensemble classification methods for classimbalance and cost-sensitive datasets. Hefei, China: University of Science and Technology of China, 2017.

[6] CHAWLA N V, LAZAREVIC A, HALL L O, et al. SMOTEBoost: improving prediction of the minority class in boosting. Proc. of the 7th European Conference on Principles and Practice of Knowledge Discovery in Databases, 2003: 107-119.

[7] FREUND Y. Experiment with a new boosting algorithm. Proc. of the 13th International Conference on Machine Learning, 1996: $148-156$.

[8] FAN W, STOLFO S J, ZHANG J. AdaCost: misclassification cost-sensitive boosting. Proc. of the 6th International Conference on Machine Learning, 1997: 97 - 105.

[9] CHAWLA N V, BOWYER K W, HALL L O, et al. SMOTE: synthetic minority over-sampling technique. Journal of Artificial Intelligence Research, 2011, 16(1): 321-357.

[10] HAN H, WANG W Y, MAO B H. Borderline-SMOTE: a new over-sampling method in imbalanced data sets learning. Proc. of the International Conference on Advances in Intelligent Computing, 2005: 878-887.

[11] ESTER M, KRIEGEL H P, SANDER J, et al. A density-based algorithm for discovering clusters in large spatial databases with noise. Proc. of the International Conference on Knowledge Discovery and Data Mining, 1996: 226-231.

[12] WASIKOWSKI M. Combating the class imbalance problem in small sample data sets. Kansas, USA: University of Kansas, 2009.

[13] JOSHI M V, KUMAR V, AGARWAL R C. Evaluating boosting algorithms to classify rare classes: comparison and improvements. Proc. of the IEEE International Conference on Data Mining, 2001: 257-264.

[14] WU G, CHANG E Y. Class-boundary alignment for imbalanced data set learning. Proc. of the Workshop on Learning from Imbalanced Data Sets, 2003: 49-56.

[15] HUANG K Z, YANG H Q, KING I, et al. Imbalanced learning with a biased minimax probability machine. IEEE Trans. on Systems, Man and Cybernetics, 2006, 36(4): 913-923.

[16] TOMEK I. Two modifications of CNN. IEEE Trans. on Systems, Man and Cybernetics, 1976, 6(11): 769-772.

[17] SÁEZ J A, LUENGO J, STEFANOWSKI J, et al. SMOTEIPF: addressing the noisy and borderline examples problem in imbalanced classification by a re-sampling method with filtering. Information Sciences, 2015, 291: 184-203.

[18] MA L, FAN S H. CURE-SMOTE algorithm and hybrid algorithm for feature selection and parameter optimization based on random forests. BMC Bioinformatics, 2017, 18: 169.

[19] DONG Y J, WANG X H. A new over-sampling approach: random-SMOTE for learning from imbalanced data sets. Proc. of the 5th International Conference on Knowledge Science, Engineering and Management, 2011: 343-352.

[20] HE H B, BAI Y, GARCIA E A, et al. ADASYN: adaptive synthetic sampling approach for imbalanced learning. Proc. of the IEEE World Congress on Computational Intelligence, 2008: $1322-1328$.

[21] BUNKHUMPORNPAT C, SINAPIROMSARAN K, LURSINSAP C. DBSMOTE: density-based synthetic minority over-sampling technique. Applied Intelligence, 2012, 36(3): $664-684$.

[22] UTIÉRREZ P D, LASTRA M, BENÍTEZ J M, et al. SMOTEGPU: big data preprocessing on commodity hardware for imbalanced classification. Progress in Artificial Intelligence, 2017, 6(4): $347-354$.

[23] ZHOU C S, LIU B, WANG S H. CMO-SMOTE: misclassification cost minimization oriented synthetic minority oversampling technique for imbalanced learning. Proc. of the 8th International Conference on Intelligent Human-Machine Systems and Cybernetics, 2016: $353-358$.

[24] ZHANG C, CHEN Y E, LIU X H, et al. Abstention-SMOTE: an over-sampling approach for imbalanced data classification. Proc. of the International Conference on Information Technology, 2017: 17-21.

[25] ZHANG Y, ZHANG H, ZHANG X, et al. Deep learning intrusion detection model based on optimized imbalanced network data. Proc. of the 18th International Conference on Communication Technology, 2018: 1128-1132.

[26] JIANG K, LU J, XIA K L. A novel algorithm for imbalance data classification based on genetic algorithm improved SMOTE. Arabian Journal for Science and Engineering, 2016, 41(8): 3255 - 3266.

[27] PRUSTY M R, JAYANTHI T, VELUSAMY K. WeightedSMOTE: a modification to SMOTE for event classification in sodium cooled fast reactors. Progress in Nuclear Energy, 2017, 100(9): $355-364$.

[28] GONG C L, GU L X. A novel SMOTE-based classification approach to online data imbalance problem. Mathematical Problems in Engineering, 2016: 5685970.

[29] XUE W, ZHANG J. Dealing with imbalanced dataset: aresampling method based on the improved SMOTE algorithm. Communications in Statistics-Simulation and Computation, 2016, 45(4): $1160-1172$.

[30] SU P H, LIU Y H, SONG X. Research on intrusion detection method based on improved smote and XGBoost. Proc. of the 8th International Conference on Communication and Network Security, 2018: 37-41.

[31] BHAGAT R C, PATIL S S. Enhanced SMOTE algorithm for classification of imbalanced big-data using random forest. Proc. of the IEEE International Advance Computing Conference, 2015: 403-408.

[32] DEMIDOVA L, KLYUEVA I. SVM classification: optimization with the SMOTE algorithm for the class imbalance prob- 
lem. Proc. of the 6th Mediterranean Conference on Embedded Computing, 2017: 1-4.

[33] JUNSOMBOON N, PHIENTHRAKUL T. Combining oversampling and under-sampling techniques for imbalance dataset. Proc. of the 9th International Conference on Machine Learning and Computing, 2017: $243-247$.

[34] GOSAIN A, SARDANA S. Farthest SMOTE: a modified SMOTE approach. Proc. of the International Conference on Computational Intelligence in Data Mining, 2017: 309-320.

[35] SUN J, LANG J, FUJITA H, et al. Imbalanced enterprise credit evaluation with DTE-SBD: decision tree ensemble based on SMOTE and bagging with differentiated sampling rates. Information Sciences, 2018, 425: 76-91.

[36] HARLIMAN K U R, UCHIDA K. Data-and algorithm-hybrid approach for imbalanced data problems in deep neural network. International Journal of Machine Learning and Computing, 2018, 8(3): 208-213.

[37] TAY F E H, SHEN L. A modified Chi2 algorithm for discretization. IEEE Trans. on Knowledge and Data Engineering, 2002, 14(3): 666-670.

[38] BAY S D. The UCI KDD repository. http://kdd.ics.uci.edu.

\section{Biographies}

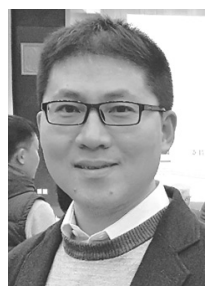

XU Xiaolong was born in 1977. He received his B.S. in computer and its applications, M.S. in computer software and theories and Ph.D. degree in communications and information systems at Nanjing University of Posts \& Telecommunications, Nanjing, China, in 1999, 2002 and 2008, respectively. He worked as a postdoctoral researcher at the Station of Electronic Science and Technology, Nanjing University of Posts \& Telecommunications from 2011 to 2013. $\mathrm{He}$ is currently a professor in College of Computer, Nanjing University of Posts \& Telecommunications. He is a senior member of China Computer Federation. His current research interests include cloud computing and big data, mobile computing, intelligent agent and information security.

E-mail:xuxl@njupt.edu.cn

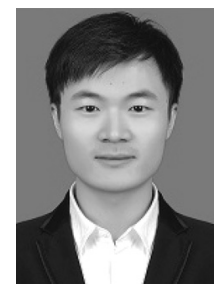

CHEN Wen was born in 1994. He received his B.E. degree in computer science and technology from Anhui Engineering University, Wuhu, China, in 2016. He works as an engineer in Institute of Big Data Research at Yancheng, Nanjing University of Posts and Telecommunications, Yancheng, China, carrying out research in data analysis.

E-mail: 1216043012@njupt.edu.cn

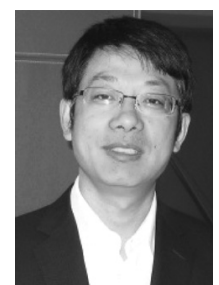

SUN Yanfei was born in 1976. He received his $\mathrm{Ph} . \mathrm{D}$. degree in communications and information systems at Nanjing University of Posts \& Telecommunications, Nanjing, China, in 2006. He is currently a professor and the director in Science and Technology Department, Nanjing University of Posts \& Telecommunications. His current research interests include communication network, mobile networks and big data.

E-mail: sunyanfei@njupt.edu.cn 